\title{
Determinación de los parámetros cinéticos de la pirolisis de la biomasa lignocelulosica
}

\section{Determination of the kinetic parameters of pyrolysis of the lignocellulosic biomass}

\author{
BARAY-GUERRERO, María del Rosario ${ }^{1} \dagger$, VALLES-ARAGON, María Cecilia ${ }^{2}$, NEVAREZ- \\ RODRIGUEZ, Myrna Concepción ${ }^{2}$ y MANJARREZ-DOMINGUEZ, Carlos Baudel ${ }^{* 2}$
}

${ }^{1}$ Universidad Autónoma de Chihuahua, Facultad de Ciencias Agrícolas y Forestales, Delicias, Chihuahua

${ }^{2}$ Universidad Autónoma de Chihuahua, Facultad de Ciencias Agro tecnológicas, Chihuahua

ID $1^{\mathrm{er}}$ Autor: María Del Rosario, Baray-Guerrero / ORC ID: 0000-0002-0289-9000, CVU CONACYT ID: 201384

ID $1^{\mathrm{er}}$ Coautor: María Cecilia, Valles-Aragon/ ORC ID: 0000-0003-1694-7634, CVU CONACYT ID: 170926

ID $2^{\text {do }}$ Coautor: Myrna Concepción, Nevarez-Rodriguez / ORC ID: 0000-0003-0325-2900, CVU CONACYT ID: 255228

ID $3{ }^{\text {er }}$ Coutor: Carlos Baudel, Manjarrez-Dominguez / ORC ID: 0000-0002-9802-7185, CVU CONACYT ID: 101794

DOI: $10.35429 /$ JRE.2020.13.4.18.25

Recibido 09 de Abril, 2020; Aceptado 30 Junio, 2020

\section{Resumen}

$\mathrm{Al}$ paso de las últimas décadas el interés por las energías renovables y el medio ambiente ha crecido considerablemente, desarrollándose un esfuerzo importante en el campo de la eficiencia energética, la creación de tecnologías sostenibles y la disminución de la huella de carbono. Es de interés, por tanto, el aprovechamiento energético de la biomasa de forma económica y eficiente, y se vuelve necesario su estudio y análisis. En este trabajo, se determinaron los parámetros cinéticos tales como, energía de activación (Ea) y factor pre-exponencial (A), fueron determinados para los residuos de la manzana usando el método termogravimétrico no-isotérmico y tratando los datos obtenidos bajo los modelos matemáticos del método diferencial y del método de la velocidad máxima, y adicionalmente realizando la distribución de energías de activación. El cálculo de la Energía de activación, ayudo a ver el modo en que transcurre la descomposición térmica (si existen uno o varios procesos y en qué rango de conversiones ocurren), a través de las constantes cinéticas características proporcionadas por los modelos cinéticos, permitió identificar las especies gaseosas emitidas por el material, y así estudiar los procesos a través de los cuales se produce tal descomposición

Parámetros cinéticos, Biomasa, Pirolisis

\begin{abstract}
Over the past decades, interest in renewable energy and the environment has grown considerably, with a significant effort being made in the field of energy efficiency, the creation of sustainable technologies and the reduction of the carbon footprint. Therefore, the use of biomass in an economical and efficient way is of interest, and its study and analysis becomes necessary. In this work, kinetic parameters such as activation energy (Ea) and preexponential factor (A) were determined for apple residues using the non-isothermal thermogravimetric method and treating the data obtained under the models mathematicians of the differential method and the maximum speed method, and additionally performing the activation energy distribution. The calculation of the activation energy helped to see the way in which thermal decomposition takes place (if there are one or more processes and in what range of conversions they occur), through the characteristic kinetic constants provided by the kinetic models, allowed to identify the gaseous species emitted by the material, and thus study the processes through which such decomposition occurs
\end{abstract}

Kinetic parameters, Biomass, Pyrolysis

Citación: BARAY-GUERRERO, María del Rosario, VALLES-ARAGON, María Cecilia, NEVAREZ-RODRIGUEZ, Myrna Concepción y MANJARREZ-DOMINGUEZ, Carlos Baudel. Determinación de los parámetros cinéticos de la pirolisis de la biomasa lignocelulosica. Revista de Energías Renovables. 2020. 4-13: 18-25

*Correspondencia al Autor (correo electrónico: rosariobaray@yahoo.com.mx)

$\dagger$ Investigador contribuyendo como primer autor. 


\section{Introducción}

El actual desarrollo económico mundial está cimentado en el comercio y la transformación del petróleo (recurso natural no renovable), no obstante, se prevé su agotamiento durante el primer cuarto del presente siglo, lo cual plantea problemas tanto económicos como de abasto de energía, ya que la demanda energética es satisfecha, principalmente, a partir de los combustibles fósiles [1]. Lograr mantener las reservas de petróleo y gas natural por un periodo mayor al estimado, mediante la sustitución gradual de las materias primas fósiles con derivados químicos y energéticos obtenidos a partir de tecnologías sustentables es una de las principales preocupaciones a nivel mundial del mundo científico.[2]

Con referencia a lo anterior los residuos agroindustriales representan una fuente renovable de energía, pues se obtienen en grandes cantidades como resultado del procesamiento industrial de frutas y hortalizas y constituyen una materia prima barata para su conversión a bio-combustibles [3] La utilización de tecnologías de energías renovables como la eólica, la geotérmica, la hidráulica, la solar y la obtenida a partir de la biomasa se presentan como alternativas en el mediano y largo plazo para el reemplazo de los combustibles fósiles [4]

Los procesos térmicos a partir de biomasa son objetivo de investigación de gran parte del mundo científico, pero sobre todo del Mundo Desarrollado, que tratan de adquirir nuevas tecnologías, amenazados por la crisis energética actual y por los problemas de contaminación ambiental [2]. La biomasa juntamente con la energía solar, representan las únicas fuentes de energía utilizadas por el hombre durante la mayor parte de la historia de la humanidad.

Los biocombustibles producidos a partir de diferentes materiales lignocelulósica como la madera, residuos agrícolas o forestales, tienen el potencial de ser un sustituto valioso, o complemento a la gasolina, los biocombustibles se refieren a combustibles líquidos o gaseosos para el sector de transporte que son predominantemente producidos por la biomasa. [4].
Los residuos agrícolas como la pomasa de la manzana (residuo sólido se compone de una mezcla compleja de cáscara, núcleo de la semilla, el cáliz, el tallo y los tejidos blandos) contienen principalmente celulosa, hemicelulosa, lignina $\mathrm{y}$ pectina. $\mathrm{Su}$ descomposición térmica revela dos regiones principales atribuidas a la descomposición de la celulosa y la hemicelulosa. La primera descomposición con pérdidas de masa que se presenta a bajas temperaturas puede asociarse al proceso de pirólisis de la hemicelulosa y a temperaturas más elevadas la pérdida en masa se asocia con descomposición de celulosa. Por otro lado, pérdidas en masa para la descomposición de lignina no se observan en este rango de temperaturas.

El conocimiento de la de volatilización de la pomasa de la manzana es importante, porque la pirólisis es el primer paso en un proceso de gasificación o combustión. [15] Los procesos de transformación de la biomasa se agrupan en tres grandes grupos: los bioquímicos, los termoquímicos, y los físico-químicos. Fundamentalmente se distinguen tres tipos de procesos termoquímicos: pirólisis, gasificación y combustión. El término pirólisis se refiere a la degradación térmica incompleta que conduce a la producción de carbón, líquidos condensables $\mathrm{y}$ alquitranes y gases.

En su sentido más estricto, la pirólisis debe realizarse en ausencia total de oxígeno, sin embargo, actualmente se utiliza este término en un sentido más amplio, para describir los cambios químicos provocados por la acción del calor [5].La pirolisis representa una nueva tecnología alternativa como procesamiento de los residuos sólidos urbanos, como una opción para el reciclado de materiales, y regenerarlos convirtiéndolos en algo provechoso para las sociedades ,en el proceso de conversión termoquímica de la biomasa es necesario conocer el mecanismo mediante el cual ocurre la degradación térmica de las diferentes fracciones que almacenan la energía química. La determinación de los parámetros cinéticos proporciona información acerca de los procesos que tienen lugar, así como de la estructura y composición de los materiales [6]. 
Además, el análisis de los productos volátiles de la degradación térmica permite identificar las especies gaseosas emitidas por el material, y así estudiar los procesos a través de los cuales se produce tal descomposición. La determinación de la cinética de descomposición de los materiales lignocelulósicos implica el conocimiento de los mecanismos de reacción. Sin embargo, el número de reacciones que ocurren simultáneamente en el proceso de pirolisis más simple es tan grande que impide desarrollar un modelo cinético que tenga en cuenta todas esas reacciones. Así, la pirolisis se estudia normalmente con modelos hipotéticos [6], en los que se considera que el comportamiento global de la pirólisis es la combinación del comportamiento de cada componente individual [6,7].

El estudio cinético trata de desvelar el modo en que transcurre la descomposición térmica (si existen uno o varios procesos y en qué rango de conversiones ocurren), a través de las constantes cinéticas características proporcionadas por los modelos cinéticos. Esto es fundamental a la hora de diseñar, construir y operar un reactor a gran escala para la pirólisis del material sujeto a estudio, para el aprovechamiento de los productos químicos que se puedan generar o para el aprovechamiento energético, o simplemente, para deshacerse de determinados materiales de una forma limpia. [6].El análisis cinético de la descomposición térmica de la biomasa se basa generalmente en la ecuación de velocidad de procesos de descomposición de sólidos [8] En el análisis termogravimétrico (TGA) de la pirólisis de materiales lignocelulósicos aparecen normalmente dos o tres picos, que pueden ser asignados a la celulosa, la hemicelulosa y la lignina, indicando por tanto, que aunque existan interacciones entre las fracciones $y$ solapamientos en su descomposición, su identidad se mantiene [6].

En éste trabajo, los parámetros cinéticos tales como, energía de activación (Ea) y factor pre-exponencial (A), fueron determinados para la biomasa residual de la manzana usando el método termogravimétrico no-isotérmico y tratando los datos obtenidos bajo los modelos matemáticos del método diferencial y del método de la velocidad máxima, y adicionalmente realizando la distribución de energías de activación.
El objetivo fue determinar los parámetros de la cinética de la pirolisis de biomasa lignocelulosica, mediante los modelos isoconversionales, así como encontrar la energía de activación representativa del proceso pirolítico.

\section{Descripción del método Materiales y Métodos}

Se emplearon muestras de residuos de manzana, procedente de los estados del Norte de Chihuahua (Cuauhtémoc, Chihuahua), este subproducto es generado en el proceso de obtención de los jugos a", el cual ha recibido muy poca atención a pesar de que posee propiedades de ser un buen combustible que produciría, energía a bajo costo.

\section{Preparación de la muestra}

El material fue sometido a un proceso secado, triturado, molido y tamizado hasta lograr los tamaños de partículas tp $<150 \mu \mathrm{m}, 180<\mathrm{tp}<250$ $\mu \mathrm{m}<425 \mu \mathrm{m}$ tp $>600 \mu \mathrm{m}$ basada en las Tablas Estándar Sieves and Mesh Sizes. El análisis inmediato que se realizo fue el Análisis porcentual en peso de los elementos para determinar Carbón, Hidrógeno, Oxígeno, Nitrógeno y Sulfuro (CHNS-O) en un analizador elemental (Carlo Erba EA 1110). El contenido de lignina, celulosa, hemicelulosa y pectina en el bagazo se determinó usando técnicas gravimétricas, descritas en la ASTM E 1756-95, D1106-95 y ASTM D1103-60.

La humedad, materia volátil y contenido de ceniza se determinarán de acuerdo al procedimiento descrito en ASTM E 871-82, ASTM 872-82 y ASTM 1755-95, respectivamente. El análisis elemental de la ceniza se realizó usando un espectrómetro de emisión atómica acoplado con plasma (AESICP). ICP Thermo Jarrel Ash, IRIS/AP DUO.Para determinar el tamaño de partícula (tp) se analizaron muestras con dimensiones de: tp $<150 \mu \mathrm{m}, 180<\mathrm{tp}<250 \mu \mathrm{m}<425 \mu \mathrm{m} \quad$ tp $>600$ $\mu \mathrm{m}$ bajo un flujo de $\mathrm{N}_{2}$ de $150 \mathrm{~cm}^{3} / \mathrm{min}$ y calentando de temperatura ambiente hasta 550 ${ }^{\circ} \mathrm{C}$ con una rapidez de $10{ }^{\circ} \mathrm{C} / \mathrm{min}$. Para verificar el efecto de la velocidad de calentamiento en la generación de materia volátil se utilizó una muestra de biomasa lignocelulósica con un mismo tamaño de partícula, que fue sometido a distintas velocidades $\left(5,10,15,20^{\circ} \mathrm{C} / \mathrm{min}\right)$. 

de Reacción.

Para la Determinación de los parámetros

La determinación de la cinética de descomposición de los materiales lignocelulósicos implica el conocimiento de los mecanismos de reacción. Sin embargo, el número de reacciones que ocurren simultáneamente en el proceso de pirolisis más simple es tan grande que impide desarrollar un modelo cinético que tenga en cuenta todas esas reacciones. Así, la pirolisis se estudia normalmente con modelos hipotéticos, en los que se considera que el comportamiento global de la pirólisis es la combinación del comportamiento de cada componente individual.

El estudio cinético trata de revelar el modo en que transcurre la descomposición térmica (si existen uno o varios procesos y en qué rango de conversiones ocurren), a través de las constantes cinéticas características proporcionadas por los modelos cinéticos. Esta información es básica cuando para diseñar, construir y operar un reactor a escala industrial para la pirolisis del material que se está estudiando, para el aprovechamiento energético de los productos que se puedan generar, con fines energéticos o para deshacerse de determinados materiales de una forma limpia.

La determinación de los parámetros cinéticos se realiza por métodos diferenciales o integrales de acuerdo al tratamiento matemático que se le dé a los datos. Entre los métodos diferenciales más usado encontramos, Freeman \& Carroll (1958), Horowitz \& Mettzger (1963), Newkirk (1960), Coats \& Redfern (1964), Vachuska \& Voboril (1971) y Friedman (1965), los cuales linealizan la ecuación diferencial y por regresión lineal se calculan los parámetros cinéticos (Freeman, et al 1965.

Este análisis cinético de la descomposición térmica de la biomasa lignocelulósica se basó en la ecuación de velocidad de procesos de descomposición de sólidos (White J. E., Catallo et al, 2011)

$$
\frac{d \alpha}{d t}=A \exp \left(\frac{-E a}{R T}\right) f(\alpha)
$$

Donde $\mathrm{t}$ denota tiempo, $\alpha$ indica la fracción de muestra que ha reaccionado o el grado de conversión, d $\alpha /$ dt es la velocidad del proceso, A y Ea son el factor pre-exponencial y la energía de activación, respectivamente, de la ecuación de Arrhenius y f ( $\alpha$ ) es una función de conversión que representa el modelo de reacción utilizado y que depende del mecanismo controlante. En este estudio el grado de conversión, $\alpha$, se ha definido como:

$\alpha=\mathrm{W}_{0}-\mathrm{W} / \mathrm{W}_{0}-\mathrm{W}$

Donde $\mathrm{w}_{\mathrm{o}}, \mathrm{w}_{\mathrm{f}} \mathrm{y} \mathrm{w}$ son las masas de muestra al inicio, final $y$ tiempo $t$, respectivamente.

Los términos desconocidos de la ecuación (1) son los parámetros de la cinética formal (A, Ea y $f(\alpha)$ ) que se utilizan para caracterizar las reacciones de pirólisis de biomasa.

Para las reacciones no-isotérmicas, donde la velocidad de calentamiento, es constante, la ecuación anterior puede expresarse como:

$\frac{d \alpha}{d t}=\beta \frac{d \alpha}{d T}=A \exp \left(\frac{-E a}{R T}\right) f(\alpha)$

Las técnicas desarrolladas para la evaluación de los parámetros cinéticos de análisis termogravimétrico no-isotermos se pueden dividir en "modelos de ajuste" y en "modelos libres". Con los "modelos libres" no es necesario asumir una cinética de reacción y los parámetros cinéticos se obtienen en función de la conversión o de la temperatura. Dentro de este tipo de modelos son los métodos isoconversionales los que asumen un grado de conversión constante y por tanto que la velocidad de reacción depende exclusivamente de la temperatura. Así, permiten estimar la energía de activación, Ea, en función de la conversión, $\alpha$, independientemente del modelo de reacción, $f(\alpha)$.

El tratamiento de datos TGA en los métodos isoconversionales puede ser diferencial o integral. En este trabajo se aplicaron un método diferencial (Friedman) y dos integrales (FlynnWall-Ozawa, Kissinger-Akahira-Sunose). El método Friedman es una técnica isoconversional diferencial, que implica tomar logaritmos naturales a cada lado de la ecuación 3 . 
$\ln \left(\frac{d \alpha}{d t}\right)=\ln \left[\beta\left(\frac{d \alpha}{d T}\right)\right]=\ln \lfloor A f(\alpha)\rfloor-\frac{-E a}{R T}$

Se asume que la función de conversión $f$ $(\alpha)$ permanece constante, lo que implica que la degradación de la biomasa es independiente de la temperatura y que depende exclusivamente de la velocidad de pérdida de masa. Una representación de $\ln (\mathrm{d} \alpha / \mathrm{d} \mathrm{t})$ versus 1/T para el mismo grado de conversión de datos tomados a varias velocidades de calentamiento dará como resultado una serie de líneas con pendientes igual a $-\mathrm{Ea} / \mathrm{R}$ para cada valor de conversión, $\alpha$, en diferentes velocidades de calentamiento $\beta$.

El método Flynn-Wall-Ozawa (FWO) es una técnica isoconversional integral donde reagrupando los términos de la ecuación, integrando éstos respecto a las variables $\alpha$ y $\mathrm{T}$ y usando la aproximación de Doyle se obtiene:

$$
\log \beta \cong \log \left(A \frac{E Q}{R g(\alpha)}\right)-2.315-0.4567 \frac{E Q}{R T}
$$

De este modo, en el método FWO, la representación de $\log \beta$ frente a $1 / \mathrm{T}$ para diferentes velocidades de calentamiento permite obtener líneas paralelas para un grado de conversión fijo. La pendiente $(-0.4567 \mathrm{Ea} / \mathrm{R}) \mathrm{de}$ esas líneas es proporcional a la energía de activación aparente. Si las Ea obtenidas son iguales para varios valores de $\alpha$, puede asumirse con certeza la existencia de una sola etapa de reacción. Por el contrario, un cambio en las Ea con el incremento del grado de conversión es indicativo de un complejo mecanismo de reacción

Otro método isoconversional integral ampliamente utilizado es el método de Kissinger-Akahira-Sunose (KAS), basado en la Ecuación (6) obtenida a partir de la aproximación de Coats- Redfern.

$\ln \left(\frac{\beta}{T^{2}}\right)=\operatorname{Ln}\left[\left(\frac{A R}{E_{a} g(\alpha)}\right)\right]-\frac{E_{a}}{R T}$

Asumiendo que $\alpha$ tiene un valor fijo, la energía de activación se puede determinar con la pendiente de la línea recta obtenida al representar $\ln \left(\beta / \mathrm{T}_{2}\right)$ versus $1 / \mathrm{T}$.

\section{Resultados}

El cálculo de la Energía de activación ayudo a ver el modo en que transcurre la descomposición térmica (si existen uno o varios procesos y en qué rango de conversiones ocurren), a través de las constantes cinéticas características proporcionadas por los modelos cinéticos. Los valores de energía de activación obtenidos en este trabajo de investigación para los modelos aplicados se muestran en las siguientes tablas de resultados

Para el Método Kissinger-AkahiraSunose (KAS) técnica isoconversional integral permitió la combinación de los métodos anteriormente mencionados (FWO y Friedman

En la Figura 1. Se asume que $\alpha$ tiene un valor fijo y que la energía de activación se puede determinar con la pendiente de la línea recta obtenida al representar $\ln \left(\beta / \mathrm{T}_{2}\right)$ versus $1 / \mathrm{T}$.

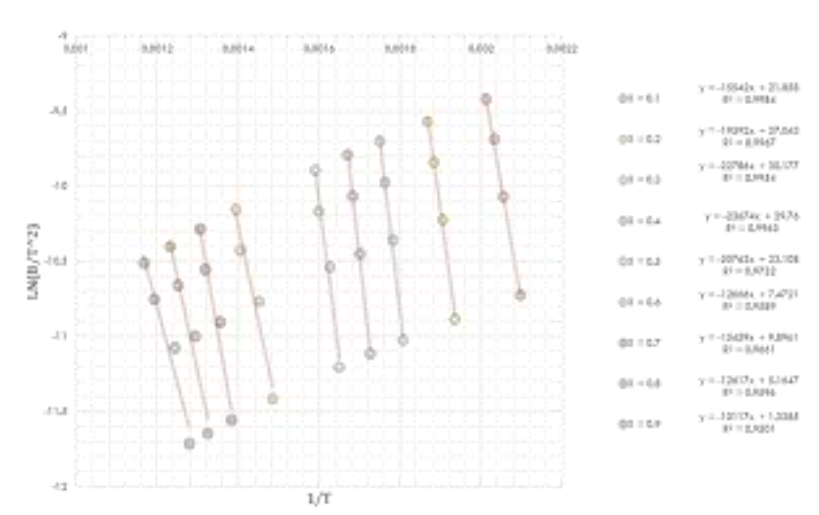

Figura 1 Metodo Kissinger-Akahira-Sunose (KAS)

La tabla 1. Muestra los resultados de los datos según el método KAS donde podemos observar el ajuste con una correlación lineal.

\begin{tabular}{|c|c|r|r|r|}
\hline \multicolumn{1}{|c|}{$\mathbf{X}$} & $\mathbf{E} / \mathbf{R}$ & $\mathbf{E}, \mathbf{k J} / \mathbf{m o l}$ & \multicolumn{1}{c|}{$\mathbf{E}, \mathbf{k c a l} / \mathbf{m o l}$} & \multicolumn{1}{c|}{$\mathbf{R}^{\wedge} \mathbf{2}$} \\
\hline 0.1 & 15542 & 129.216188 & 30.8826689 & 0.9984 \\
\hline 0.2 & 19592 & 162.887888 & 38.9302052 & 0.9967 \\
\hline 0.3 & 22786 & 189.442804 & 45.2768302 & 0.9954 \\
\hline 0.4 & 23674 & 196.825636 & 47.041327 & 0.9963 \\
\hline 0.5 & 20763 & 172.623582 & 41.2570361 & 0.9732 \\
\hline 0.6 & 12666 & 105.305124 & 25.1679246 & 0.9589 \\
\hline 0.7 & 15459 & 128.526126 & 30.7177441 & 0.661 \\
\hline 0.8 & 12617 & 104.897738 & 25.0705594 & 0.9596 \\
\hline 0.9 & 10117 & 84.112738 & 20.1029444 & 0.9501 \\
\hline
\end{tabular}

Tabla 1 Resultados con el Método Kissinger-AkahiraSunose (KAS) 
De acuerdo al Modelo Friedman como es una técnica isoconversional diferencial, que implica tomar logaritmos naturales a cada lado de la ecuación la energía de activación se puede calcular con la siguiente ecuación Permitiendo que la función de conversión $\mathrm{f}(\alpha)$ permanece constante, lo que implica que la degradación de la biomasa es independiente de la temperatura y que depende exclusivamente de la velocidad de la pérdida de la masa. En la figura 2 se muestra las $E_{a} / R$ en función de las conversiones $f(\alpha)$ desde 0.1 hasta 0.9 .

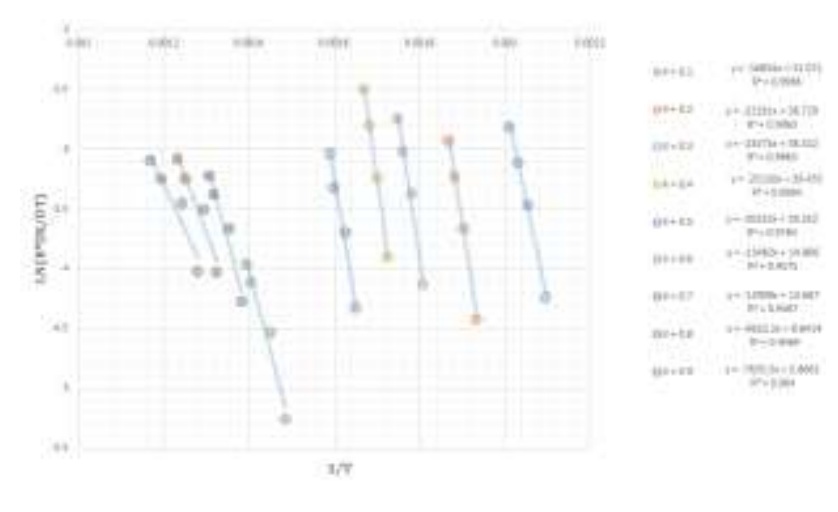

Figura 2 Método Friedman

En la tabla 2 se muestran los valores de las Energía de Activación en relación con el factor pre-exponencial

\begin{tabular}{|r|r|r|r|r|}
\hline $\mathbf{X}$ & \multicolumn{1}{|c|}{$\mathbf{E} / \mathbf{R}$} & $\mathbf{E}, \mathbf{k J} / \mathbf{m o l}$ & $\mathbf{E}, \mathbf{~ k c a l} / \mathbf{m o l}$ & \multicolumn{1}{c|}{$\mathbf{R}^{\wedge} \mathbf{2}$} \\
\hline $\mathbf{0 . 1}$ & 16834 & 139.957876 & 33.4499324 & 0.9966 \\
\hline $\mathbf{0 . 2}$ & 22291 & 185.327374 & 44.2932424 & 0.9963 \\
\hline $\mathbf{0 . 3}$ & 23575 & 196.00255 & 46.8446095 & 0.9863 \\
\hline $\mathbf{0 . 4}$ & 25120 & 208.84768 & 49.9145955 & 0.9994 \\
\hline $\mathbf{0 . 5}$ & 20323 & 168.965422 & 40.3827359 & 0.9764 \\
\hline $\mathbf{0 . 6}$ & 13482 & 112.089348 & 26.7893542 & 0.9575 \\
\hline $\mathbf{0 . 7}$ & 12909 & 107.325426 & 25.6507768 & 0.9467 \\
\hline $\mathbf{0 . 8}$ & 9652.2 & 80.2483908 & 19.1793654 & 0.9469 \\
\hline $\mathbf{0 . 9}$ & 7635.5 & 63.481547 & 15.1720897 & 0.904 \\
\hline
\end{tabular}

Tabla 2 Resultados con Método Friedman

Con Método Flynn-Wall-Ozawa (FWO) se comprueba que mediante esta técnica isoconversional integral donde Integrando éstos respecto a las variables $\alpha$ y $\mathrm{T}$ y usando la aproximación de Doyle se obtiene

$$
\log \beta \equiv \log \left(A \frac{E_{a}}{R g(\alpha)}\right)-2,315-0,4567 \frac{E_{a}}{R T}
$$

La Figura 3 muestra los gráficos lineales donde se observa la representación de $\log \beta$ frente a $1 / \mathrm{T}$ para diferentes velocidades de calentamiento que permitió obtener líneas más paralelas para un grado de conversión con un valor de $-0.453 \mathrm{Ea} / \mathrm{R}$ este método es el que mejor ajusta los datos

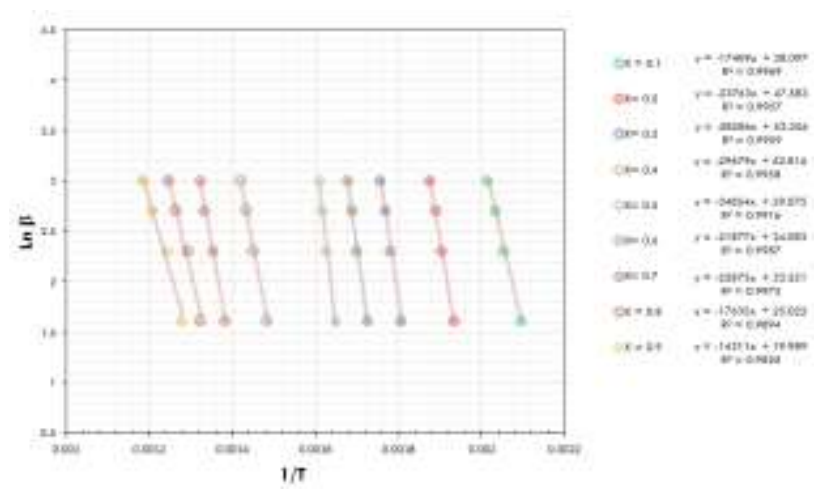

Figura 3 Con Método Flynn-Wall-Ozawa (FWO)

\begin{tabular}{|c|r|r|r|r|}
\hline $\mathbf{X}$ & \multicolumn{1}{c}{ E/R } & \multicolumn{1}{c}{ E, kJ/mol } & \multicolumn{1}{c|}{ E, kcal/mol } & \multicolumn{1}{c|}{$\mathbf{R}^{\wedge} \mathbf{2}$} \\
\hline $\mathbf{0 . 1}$ & 973.34 & 8.09234876 & 1.93407135 & 1 \\
\hline $\mathbf{0 . 2}$ & 1051.7 & 8.7438338 & 2.08977628 & 1 \\
\hline $\mathbf{0 . 3}$ & 1124.7 & 9.3507558 & 2.23483064 & 1 \\
\hline $\mathbf{0 . 4}$ & 1177.7 & 9.7913978 & 2.34014407 & 1 \\
\hline $\mathbf{0 . 5}$ & 1234.8 & 10.2661272 & 2.4536044 & 1 \\
\hline $\mathbf{0 . 6}$ & 1390.4 & 11.5597856 & 2.76278876 & 0.9999 \\
\hline $\mathbf{0 . 7}$ & 1488.2 & 12.3728948 & 2.95712186 & 1 \\
\hline $\mathbf{0 . 8}$ & 1565.1 & 13.0122414 & 3.10992569 & 0.9999 \\
\hline $\mathbf{0 . 9}$ & 1635.7 & 13.5992098 & 3.25021114 & 0.9999 \\
\hline
\end{tabular}

Tabla 3 Resultados con Método Flynn-Wall-Ozawa (FWO)

Huang y col. analizan el desarrollo de los valores de energía de activación con el grado de conversión, encontrando valores más bajos para conversiones menores, un crecimiento de éstos en torno a conversiones intermedias, para volver a bajar al final del proceso. Estos autores han relacionado este comportamiento con la descomposición de las fracciones (hemicelulosa, celulosa y lignina) ya que los valores de $E a$ obtenidos en esos rangos de conversión son cercanos a los valores tabulados de estos compuestos puros.

Las energías de activación calculadas para métodos Friedman, FWO y KAS se presentan en la tabla 12. Las energías de activación media calculada a partir de los métodos de Friedman, FWO y KAS fueron $197,7,213,0$ y $201,7 \mathrm{~kJ} / \mathrm{mol}$, respectivamente. Se muestra también, excelentes coeficientes de correlación lineal se obtuvieron con un $\mathrm{R}^{2}$ muy cerca de 1 con 0,973, 0,996 y 0,978, para el Friedman, OFW y métodos KAS respectivamente. Resultados obtenidos a partir de todos los modelos estaban en un buen acuerdo con una desviación por debajo del $8 \%$. 
Las pequeñas desviaciones de la energía de activación alta (FWO) con respecto a los métodos de Friedman y KAS fueron del 7,1 y $5,6 \%$, respectivamente, lo que valida la fiabilidad de los cálculos y confirmó el poder predictivo de los métodos KAS y FWO.

\begin{tabular}{|r|r|r|r|}
$\begin{array}{c}\text { Conversión } \\
(\mathbf{x})\end{array}$ & \multicolumn{1}{c}{$\begin{array}{c}\text { KAS, } \\
\text { KJ/mol }\end{array}$} & $\begin{array}{c}\text { FWO, } \\
\text { KJ/mol }\end{array}$ & $\begin{array}{c}\text { Friedman, } \\
\text { KJ/mol }\end{array}$ \\
\hline 0.1 & 137 & 145 & 148 \\
\hline 0.2 & 189 & 198 & 213 \\
\hline 0.3 & 228 & 238 & 235 \\
\hline 0.4 & 237 & 247 & 249 \\
\hline 0.5 & 280 & 290 & 267 \\
\hline 0.6 & 170 & 182 & 175 \\
\hline 0.7 & 134 & 189 & 145 \\
\hline 0.8 & 174 & 147 & 100 \\
\hline 0.9 & 105 & 119 & 78 \\
\hline
\end{tabular}

Tabla 4 Valores de Energía de Activación determinado por los métodos Friedman, Flynn-Wall-Ozawa, KissingerAkahira-Sunose

La figura 4. Muestra que los 3 modelos siguen la misma tendencia de la Ea con respecto al factor de conversión $\alpha=0,49$ se demuestra que la Ea si depende altamente de la conversión que significa que el bagazo de pirólisis pulpa de manzana es un proceso complejo que consta de varias reacciones Cuando es mayor la Ea más lenta es la reacción

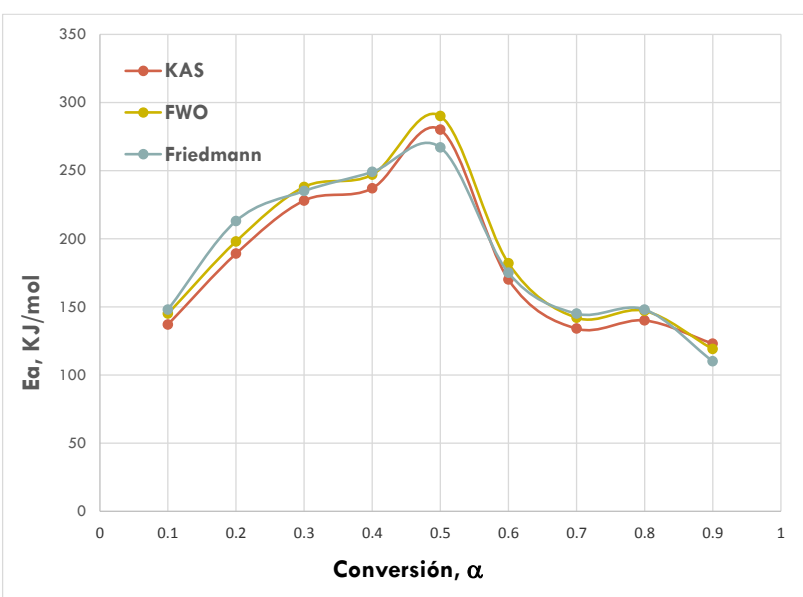

Figura 4 Valores de Energía de Activación determinado por los métodos Friedman, Flynn-Wall-Ozawa, KissingerAkahira-Sunose

\section{Conclusiones}

Los valores de energía de activación obtenidos en la presente investigación para los modelos aplicados están dentro del rango de valores de energía de activación de la hemicelulosa (67 a $105 \mathrm{~kJ} / \mathrm{mol}$ ), celulosa (210 a $240 \mathrm{~kJ} / \mathrm{mol}) \mathrm{y}$ lignina (65 a $67 \mathrm{~kJ} / \mathrm{mol})$.
La energía de activación $\left(\mathrm{E}_{\mathrm{a}}\right)$ que se ajusta mejor a los datos experimentales fue de $213.0 \mathrm{~kJ} / \mathrm{mol}$, obtenido por el modelo FWO Huang et al., han estudiado la evolución de los valores de energía de activación como una función del grado de conversión, la búsqueda de bajos valores de Ea para pequeñas conversiones, un crecimiento de estos en las conversiones intermedias para volver a valores bajos hacia el final.

Estos autores han relacionado este comportamiento a la descomposición de las fracciones de hemicelulosa, celulosa y lignina. Puesto que, los valores de Ea obtenidos en estos rangos de conversión están cerca de los valores tabulados de los compuestos puros. Sin embargo, hay que señalar que los valores de energía de activación que se determinan para cualquier valor de conversión no deben ser considerados como los valores reales de una etapa de reacción particular, sino como un valor aparente que representa las contribuciones de numerosas reacciones paralelas y que compiten, que contribuyen a la velocidad de reacción global. Para un proceso de desvolatilización biomasa tan complejo, las contribuciones variarán con la temperatura y la conversión y muy a menudo se superponen unos a otros

\section{Referencias}

[1] El Bassam, N. 2001. Renewable energy for rural communities. Renew Energ 24(3-4): 401408

[2] (Hong y col., 2009; Suiran y Jing, 2009).

[3] Hong, Y., Yuan Z., Junguo, L. 2009. Land and water requirements of biofuel and implications for food sup-ply and the environment in China. Energ Policy 37(5):18761885.

[4] I.D.A.E.- Instituto para la Diversificación y el Ahorro de Energía. biomasa.Disponible en la web:

http://www.idae.es/index.php/idpag.233/relcate goria.1037/ relmenu.48/mod.pags/mem.detalle

[5] Juan A. Conesa Ferrer. Curso Básico de Análisis Térmico. Termogravimetria, cinética de reacciones y análisis térmico diferencial. Universidad de Alicante, Mayo 2000. 
[6] Juan A. Conesa, A. Domene. Biomasses pyrolysis and combustion kinetics through $n$-th order parallel reactions. Thermochimica Acta, Volume 523, Issues 1-2, Pages 176- 181, August 2011

[7] Agustín García Barneto, José Ariza Carmona, José Enrique Martín Alfonso, Jesús Díaz Blanco. Kinetic models based in biomass components for the combustion and pyrolysis of sewage sludge and its compost. Journal of Analytical and Applied Pyrolysis, Volume 86, Issue 1, pp. 108-114, September 2009

[8] Fan-fei MIN, Ming-xu ZHANG, Qing-ru CHEN. Non-isothermal Kinetics of Pyrolysis of Three Kinds of Fresh Biomass. Journal of China University of Mining and Technology, Volume 17, Issue 1, pp. 105-111, March 2007.

[9] IEA - International Energy Agency. Clean Energy Progress Report. IEA input to the Clean Energy Ministerial. Disponible online en la web: http://www.iea.org/papers/2011/CEM_Progress _Report.pdf.

[10] Friedman H. Kinetics of thermal degradation of char-forming plastics from thermogravimetry. Application to a phenolic plastic. Journal of Polymer Science Part C: Polymer Symposia, Volume 6, Issue 1, pp. 183195, 1964.

[11] Ozawa T. A new method of analyzing thermogravimetric data. Bulletin of the Chemical Society of Japan, 38, pp.1881-6, 1965.

[12] Kissinger HE. Reaction kinetics in differential thermal analysis. Analytical Chemitry, 29, pp.1702-6, 1957.

[13] T.Akahira and T. Sunose: Trans. Joint Convention of Four Electrical Institutes, paper $\mathrm{n}^{\circ}$ 246, 1969.

[14] AGENEX - Agencia Extrema de la Energía. Los residuos de industrias agrícolas.

[15] Muller, M.; Bockhorn, H.; Krebs, L.; Muller, U.; J. Anal. Appl. pyrolysis 2003, 68, 231.
[16] Young, H.; Young, P.; Jinsoo, K.; Seung, S.; Seung, K.; Bioresour. Technol. 2009, 100, 400

[17] Agustín García Barneto, José Ariza Carmona, José Enrique Martín Alfonso, Jesús Díaz Blanco. Kinetic models based in biomass components for the combustion and pyrolysis of sewage sludge and its compost . Journal of Analytical and Applied Pyrolysis, Volume 86, Issue 1, pp. 108-114, September 2009

[18] Shuyuan, L.; Changtao, Y.; Fuel Process. Technol. 2003, 85, 51.

[19] Suuberg, E.; Combust. Flame 1983, 50, 243

[20] Teng, H.; Hsieh, C. T.; Ind. Eng. Chem. Res. 1999, 38, 292.

[21] Du, Z. Y.; Sarofim, A. F.; Longwell, J. P.; Energy Fuels 1990, 4, 296

[22] Alexander, G.; Wolfgang, K.; Sonia, R.; Tesis de Doctorado, Universidad Nacional de Colombia, Colombia y Universidad de Kassel, Alemania, 2008.

[23] Xiaodong, Z.; Min, X.; Rongfeng, S.; Sun, 1.; J. Eng. Gas Turbines 2006, 128, 493. 